\title{
When an Infant Dies: The Need to Acknowledge Grief and Find Bereavement Support
}

Barb Himes, IBCLC

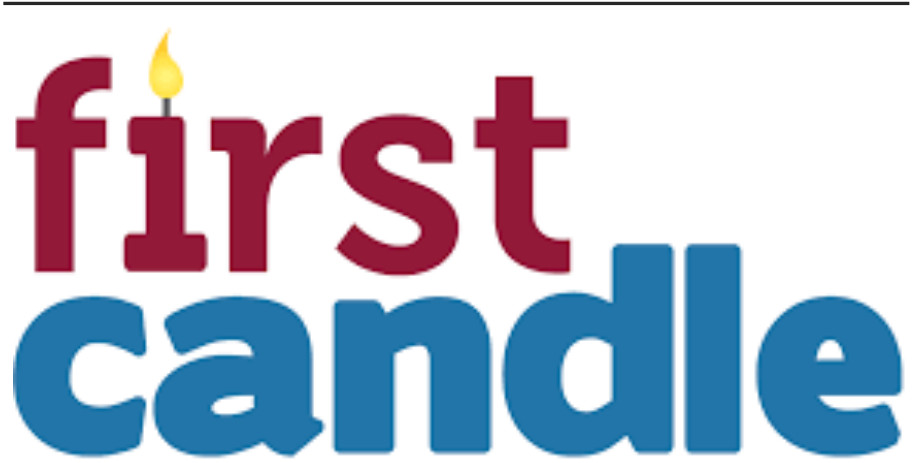

Saving babies. Supporting families.

First Candle's efforts to support families during their most difficult times and provide new answers to help other families avoid the tragedy of the loss of their baby are without parallel.

We are in a time when pandemic conditions have placed an additional burden on grief, not only because coronavirus brings its own paths of mortality with it, but because it complicates feelings of grief and bereavement even when the losses are not related to it.

\section{"We are in a time when pandemic conditions have placed an additional burden on grief, not only because coronavirus brings its own paths of mortality with it, but because it complicates feelings of grief and bereavement even when the losses are not related to it."}

First Candle's mission is the elimination of Sudden Infant Death Syndrome (SIDS) and other sleep-related infant deaths, through education, while providing support for grieving families who have suffered a loss.

This bereavement support takes several forms and specifically assists different causes of infant loss:

- A 24-hour grief line. Grief counseling day or night for a family member in need, offering one-on-one support and access to materials and local support services.

- $\quad$ Bereavement library. Resources on surviving the death of a baby, guidance on coping with birthdays, anniversaries, holidays, and difficult considerations such as having another child, as well as ways for families to honor the memory of their baby.

- $\quad$ Peer-to-peer online support groups. A safe and supportive environment for individuals and families to talk about pregnancy and infant loss. Group members connect and find an empathetic outlet for their feelings of grief, anger, anxiety, and depression, sharing personal experiences and offering one another emotional comfort and moral support. There are separate groups that specifically address infant loss due to SIDS and other sleep-related causes, stillbirth, and miscarriage.

We are finding that the nature of this Covid-19 time can add complexity to the already painful burden of infant loss and that grief itself can widen, as we hear not only from families suffering an immediate loss but also from those who are re-experiencing grief. It may be for an infant who died years past, and the memory of that loss is being rekindled in this period of global morbidity and mortality, or it may even be a feeling of grief for another lost family member that is even heavier now.

It brings to light the reality that there is a patchwork of existing bereavement support services that is further diminished - such as restriction or unavailability of in-person group gatherings - during this pandemic, and underscores the value of those services that do exist. People will turn to what they can find in their time of need.

It also reminds us that experiencing infant loss and addressing grief can be part of the prenatal, perinatal and postpartum health care professional's environment, presenting difficulties for both the grieving family seeking counsel and support and for the health professionals themselves, as their overriding drive is at all times to save the infant and maternal life. The focus is on survival, not death, and dying.

So there may, therefore, be times when simple, straightforward

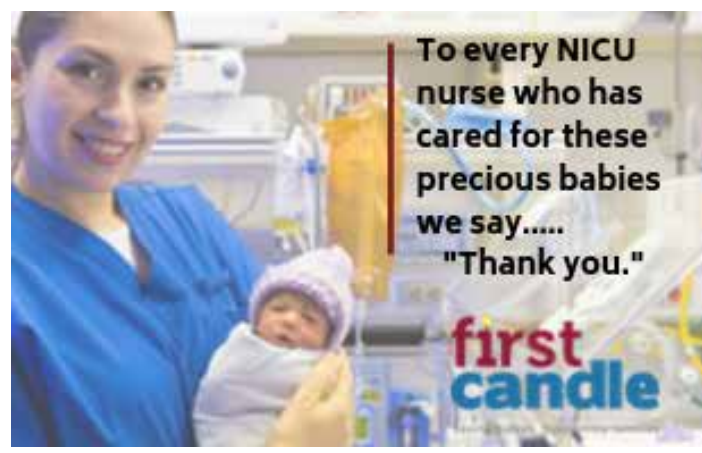

\section{Did you know that premature and low birth weight babies have a $4 \times$ greater risk for SIDS?}

At First Candle we're educating parents, grandparents and caregivers about safer sleep to make sure all babies reach their first birthday. Learn more at firstcandle.org 
guidance around bereavement situations can be helpful. For hospitals, this can include:

- $\quad$ Listen. The number one way to support a grieving person is to let them talk and then listen.

- Recognize. Say the baby's name.

- Be open and sincere. "I'm sorry." "I can't imagine what you are going through." "I am here for you."

- $\quad$ Avoid cliché. "At least you have your other children." "God needed another angel." "You can have another." "It's God's will."

- $\quad$ Offer keepsakes. A lock of hair; foot and handprints; pictures.

- Explain. Share what will happen next (autopsy, funeral home, coroner's office).

- Identify grief support resources. This could include the hospital chaplain, First Candle's bereavement support services, or local support groups.

For physicians, this can also mean offering to review the infant's autopsy results and counseling the parents to look after themselves, so that they may continue to provide for each other and the rest of the family. This does not, however, mean pushing grief aside in order to appear strong, another area bereavement support can help both mothers and fathers address.

\section{"For physicians, this can also mean}

offering to review the infant's autopsy results and counseling the parents to look after themselves, so that they may continue to provide for each other and the rest of the family."

This last point also matters for professionals. We know from experience that when the intense daily focus is on saving babies' lives, and one is lost, there can be a debilitating emotional impact on providers, making it important for them to identify resources for their own support as well as for their patients.

As we discuss in our support programs, infant death can unleash a range of emotions:

- Guilt. Self-blame, founded or unfounded.

- $\quad$ Anger. Self-directed or at others.

- $\quad$ Fear. An overall sense of dread, which can affect daily living and interpersonal relationships.

- Depression. Episodes of fatigue, sadness, feelings of worthlessness.
All of these may present themselves in unique ways from individual to individual, and support services should work to give parents a caring hand in their pain and also help them to work their personal way through the grieving process and emerge into resolution and recovery. Grief is an intense, lonely and personal experience, and for parents, it can be further affected by whether this is their first experience with death, whether they have other children or may consider having them in the future, and how they will incorporate the memory of this infant in their lives going forward. The grief may be so intense that they find themselves struggling for ways to relate to each other as well as to their friends and relatives.

For health care professionals, it is the awareness that the families will need immediate attention to their grief and that they themselves may not be immune to the effects of infant loss.

All parties need to be caring for themselves.

Information on First Candle and its bereavement support services can be found on the First Candle website. The Grief Support Line is $1-800-221-7437$.

\section{References:}

1. Surviving the Death of a Baby: Grief Resources from First Candle. https://firstcandle.org/wp-content/uploads/2018/04/Surviving-the-Death-of-a-Baby.pdf

Disclosure: The author is the Director of Education and Bereavement Services of First Candle, Inc., a Connecticut not for profit 501c3 corporation.

NT

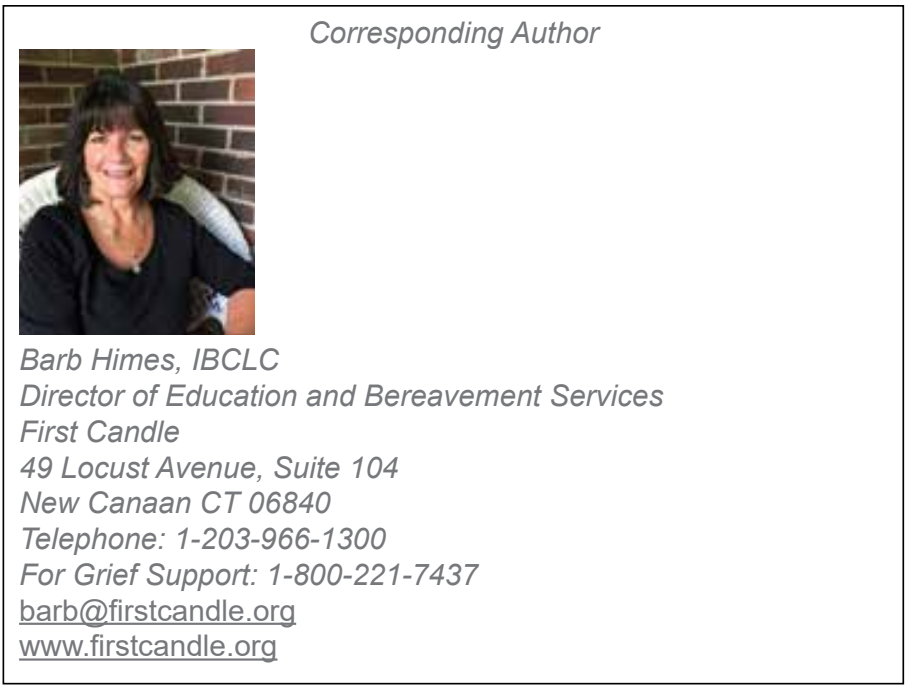

